\title{
Communication \\ Generation of Ultrabroad and Intense Supercontinuum in Mixed Multiple Thin Plates
}

\author{
Jing Li, Wenjiang Tan *, Jinhai Si, Zhen Kang and Xun Hou
}

Key Laboratory for Physical Electronics and Devices of the Ministry of Education, Shaanxi Key Lab of Information Photonic Technique, School of Electronic Science and Engineering, $\mathrm{Xi}^{\prime}$ an Jiaotong University, Xi'an 710049, China; lijing525@stu.xjtu.edu.cn (J.L.); jinhaisi@mail.xjtu.edu.cn (J.S.);

kangzhen@stu.xjtu.edu.cn (Z.K.); houxun@mail.xjtu.edu.cn (X.H.)

* Correspondence: tanwenjiang@mail.xjtu.edu.cn

\section{check for} updates

Citation: Li, J.; Tan, W.; Si, J.; Kang, Z.; Hou, X. Generation of Ultrabroad and Intense Supercontinuum in Mixed Multiple Thin Plates. Photonics 2021, 8, 311. https://doi.org/ $10.3390 /$ photonics 8080311

Received: 21 June 2021

Accepted: 27 July 2021

Published: 3 August 2021

Publisher's Note: MDPI stays neutral with regard to jurisdictional claims in published maps and institutional affiliations.

Copyright: (c) 2021 by the authors. Licensee MDPI, Basel, Switzerland. This article is an open access article distributed under the terms and conditions of the Creative Commons Attribution (CC BY) license (https:// creativecommons.org/licenses/by/ $4.0 /)$.

\begin{abstract}
Supercontinuum (SC) generation using multiple thin plates is demonstrated with a femtosecond laser pulse. We propose an improved technique to obtain larger spectrum broadening and higher spectral intensity by employing mixed multiple thin plates with different thicknesses and materials. Furthermore, the spectrum has good stability, which is superior to that of the spectrum induced by the traditional single filament in bulk material. Our approach offers a route towards simple and stable SC generation for potential applications.
\end{abstract}

Keywords: femtosecond laser; supercontinuum generation; spectral broadening; multiple thin plates

\section{Introduction}

SC generation is a universal phenomenon produced by the nonlinear propagation of intense femtosecond laser pulses in a transparent medium [1]. SC is characterized by an extremely broad spectrum with frequencies ranging from infrared to ultraviolet. The underlying physics of filament-induced SC generation appears to be a complex process that involves a dynamic interplay of spatial and temporal effects: self-focusing, self-phase modulation, self-steepening, group velocity dispersion, and multiphoton absorption [2-6]. SC has attracted significant research interest in diverse fields owing to its advantages of ultrabroadband radiation, high spectral brightness, and high spatial coherence, which are equivalent to those of white-light lasers. It has found wide application in optical frequency combs [7], biomedical imaging [8], molecular fingerprint spectroscopy [9], and the generation of few-cycle femtosecond pulses [10].

In recent years, there have been many ways to generate an ultrabroadband SC. For instance, a femtosecond laser pulse is injected into inert-gas-filled hollow-core fibers. This method could enable the compression of pulses with high compression ratios and better spatial modes [11,12]. However, it has some inherent drawbacks, such as low efficiency of fiber coupling and complexity of adjustment of the experimental apparatus. In addition, SC can be efficiently generated in a solid-state material due to its higher nonlinear index of refraction. It is a simple and robust method to broaden the spectrum. However, when the peak power of the laser pulse is sufficiently high, self-focusing and multiphoton ionization result in optical breakdown and permanent damage to the material. More recently, a novel technique that generates SCs with multiple thin plates has attracted much attention [13-16]. This technique effectively circumvents energy loss and optical breakdown. It has been reported in nearly one octave-spanning spectrum ranging and a few-millijoule pulse energy $[17,18]$. Different applications place differing demands on the characteristics of the SC spectrum. For example, the spectral coherence and a broad spectral range are the key factors in pulse compression. In the application of femtosecond transient absorption spectroscopy, high intensity of shortwave components will provide a broad spectral detection range, and better spectral stability will improve the detection sensitivity. 
In addition to supercontinuum generation, the use of thin plates makes it possible to perform more complex manipulations with high-energy ultrashort pulses. A new concept of using thin plates was proposed for spectral broadening with subsequent temporal compression of ultrashort pulses having energy as high as a few hundred Joules [19]. An experiment was reported in which the successful compression of high-energy laser pulses was achieved [20]. The measured spectrum broadening in thin plates together with the fundamental spectrum also allows the reconstruction of the pulse intensity and phase [21].

In this paper, we experimentally investigated the generation of SC by mixing multiple thin plates. The broadening and intensity of the SC spectrum in the short-wavelength region was further enhanced through a set of multiple thin plates with different thicknesses and materials.

\section{Experiments}

The experimental apparatus of SC generation in multiple thin plates is illustrated in Figure 1. A Ti:sapphire femtosecond laser system operating with a pulse duration of $50 \mathrm{fs}$ and central wavelength of $800 \mathrm{~nm}$ at a repetition rate of $1 \mathrm{kHz}$ was used in our experiment. A neutral density filter placed in the beginning was used to control the average power of the laser beam to obtain a suitable energy per pulse of $520 \mu \mathrm{J}$. The beam diameter was approximately $10 \mathrm{~mm}$. The output pulse was loosely focused onto a set of fused silica thin plates with a convex lens $(\mathrm{f}=2000 \mathrm{~mm})$. The diameter of the focal point was approximately $410 \mu \mathrm{m}$ at the $1 / \mathrm{e}^{2}$ width measured by the knife-edge method. The peak intensity was approximately $7.9 \times 10^{12} \mathrm{~W} / \mathrm{cm}^{2}$ at the focal point. The fused silica thin plates (Beijing Zhong Cheng Quartz Glass Co., Ltd., Beijing, China) used in our paper were squares with sides of $20 \mathrm{~mm}$ and thicknesses of $120 \mu \mathrm{m}$ and $200 \mu \mathrm{m}$. These plates were placed at the waist of the beam at the Brewster angle $\left(55.5^{\circ}\right)$ to reduce the Fresnel reflection loss at the surfaces. The generated SC spectrum was collected by a lens on a fiber-coupled spectrometer (Ocean Optics HR 4000). A filter was used in front of the spectrometer to suppress the fundamental frequency light at a center wavelength of $800 \mathrm{~nm}$.

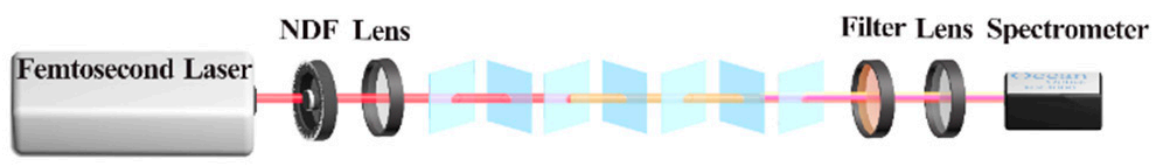

Figure 1. Schematic of the experimental setup (NDF: neutral density filter).

\section{Results and Discussion}

Firstly, seven $120 \mu \mathrm{m}$ thin fused silica plates were applied for SC generation. The first thin plate was placed $20 \mathrm{~cm}$ before the focus of the lens, and the average power was approximately $489 \mathrm{~mW}$ after the plate. The spacing between these thin plates was $8 \mathrm{~cm}$, $10 \mathrm{~cm}, 5 \mathrm{~cm}, 2.5 \mathrm{~cm}, 2.5 \mathrm{~cm}, 2.5 \mathrm{~cm}$, respectively. The positions of the plates were determined by obtaining the broadest transmitted spectrum after each plate without optical damage. The thin plates should be placed in sequence, resulting in the best spectrum broadening. As the laser passes through the thin plate, the self-focusing effect causes the laser beam to form a focal point in the air. It should be noted that the next thin plate should be placed away from the focal point to effectively avoid material damage. The beam diameter was increased after the seventh plate, leading to a reduction in peak power. When more $120-\mu \mathrm{m}$-thin fused silica plates were added, the width of the spectrum ceased to broaden. The average power after the second to seventh plates was $480 \mathrm{~mW}, 473 \mathrm{~mW}, 458 \mathrm{~mW}, 448 \mathrm{~mW}, 437 \mathrm{~mW}$, and $428 \mathrm{~mW}$, respectively. To understand the process of spectrum broadening, the fundamental spectrum and the SC spectrum after each plate were recorded, as shown in Figure 2. It was observed that the broadened spectra in the first three plates were symmetric to the fundamental spectrum, which coincided with the process of self-phase modulation. However, the spectra were significantly broadened from the fourth to seventh thin plates, as shown in Figure $2 \mathrm{~b}$. The primary mechanism responsible for spectral broadening was the nonlinear phase accumulation pass through the plate, leading to pulse self-steepening. 
As a comparison, the supercontinnum generation in an $840 \mu \mathrm{m}$ fused silica plate without filamentation was also investigated. The thickness of this plate was equivalent to the sum of the seven thin plates mentioned above. To avoid the filamentation, this single plate was placed $25 \mathrm{~cm}$ before the focal point, and the SC spectrum is shown by the red dotted line in Figure 2b. The output average power was approximately $455 \mathrm{~mW}$. The spectral width induced by the single plate was narrower than that of the multiple thin plates due to the lower input laser intensity.

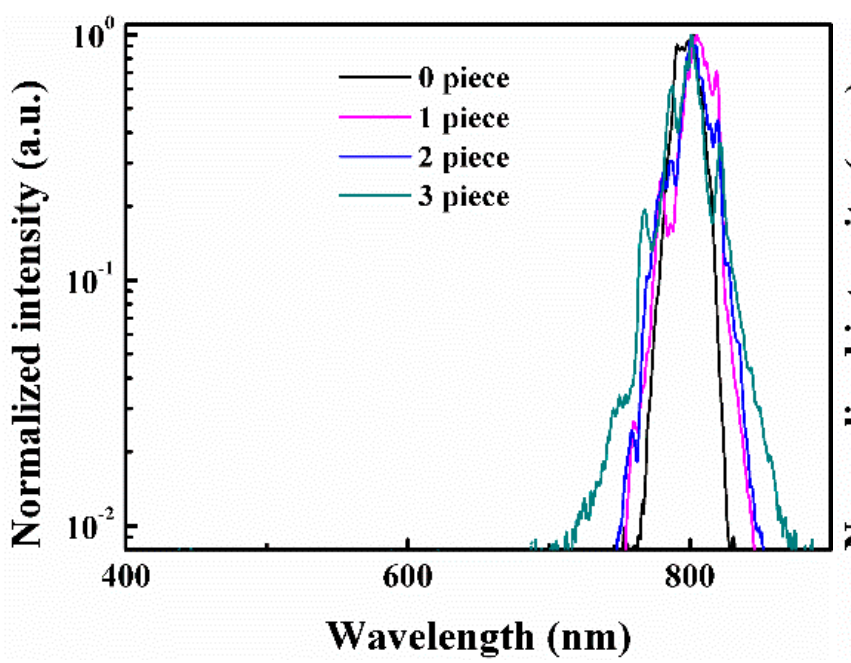

(a)

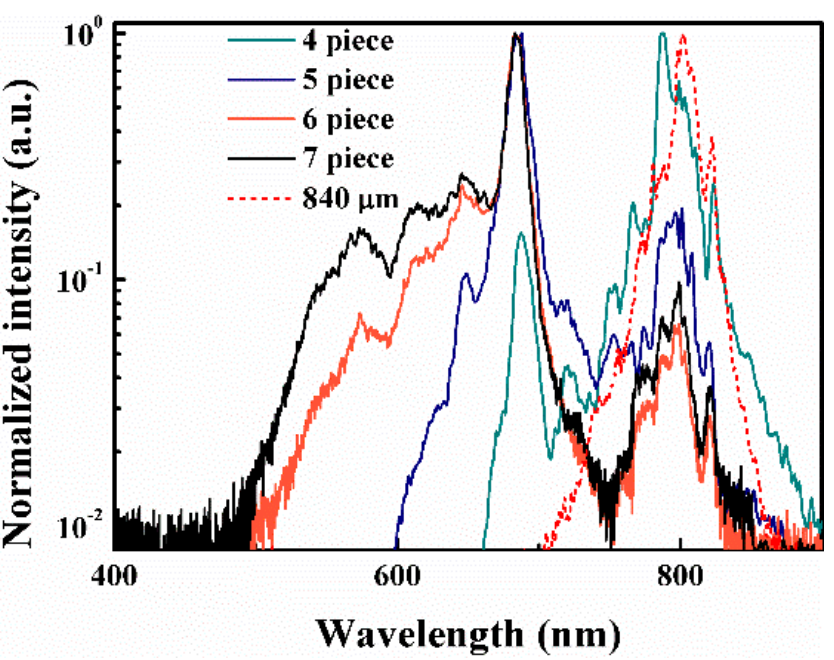

(b)

Figure 2. Spectra passing through the fused silica plates. The " 0 piece" indicates fundamental spectrum. Red dotted line represents the spectrum generated from the single $840 \mu \mathrm{m}$ plate. Each spectrum was normalized by its maximum value.

We compared the effect of different thicknesses in multiple thin plates on spectral broadening. The spectrum of SC generation with $120 \mu \mathrm{m}$ and $200 \mu \mathrm{m}$ thin fused silica plates is shown in Figure 3. Seven plates with the same thickness were strategically placed at the waist position of the incident laser beam. It was observed that a broader spectrum could be obtained with seven pieces of $120 \mu \mathrm{m}$ thin fused silica plates, and the final spectrum extended to approximately $490 \mathrm{~nm}$ in the short-wavelength region. However, the cutoff wavelength in the short-wavelength region was approximately $520 \mathrm{~nm}$ for $200 \mu \mathrm{m}$ thin plates. In the course of the experiment, it was found that $200 \mu \mathrm{m}$ thin plates were easily damaged and had poor stability. Essentially, there was a longer optical path inside the thicker plates, which was more prone to energy loss from multiphoton ionization and optical damage, resulting in less spectral broadening. However, a thinner plate has a shorter optical path, which avoids these disadvantageous factors but provides very limited self-phase modulation. Self-phase modulation is usually characterized by the B-integral. It is defined as $B=\frac{2 \pi}{\lambda} \int_{0}^{L} n_{2} I d z$, where $\lambda$ is the central wavelength, $n_{2}$ is the nonlinear refraction index, $I$ is the intensity of incident light, $z$ is the coordinate along the beam direction, and $L$ is the thickness of Kerr medium. When the optical intensity is constant, the B-integral increases with the propagation distance. The nonlinear refractive index for fused silica is $2.4 \times 10^{-16} \mathrm{~cm}^{2} / \mathrm{W}$. The estimated value of the B-integral is approximately 3.0 for the $200 \mu \mathrm{m}$ thin plate and 1.8 for the $120 \mu \mathrm{m}$ thin plate in our experiment. Thus, the accumulation of self-phase modulation for the $200 \mu \mathrm{m}$ thin plate is 1.67 times that of the $120 \mu \mathrm{m}$ plate. We infer that if a set of thin plates with different thicknesses was employed, the SC spectrum would have a more expanded range. 


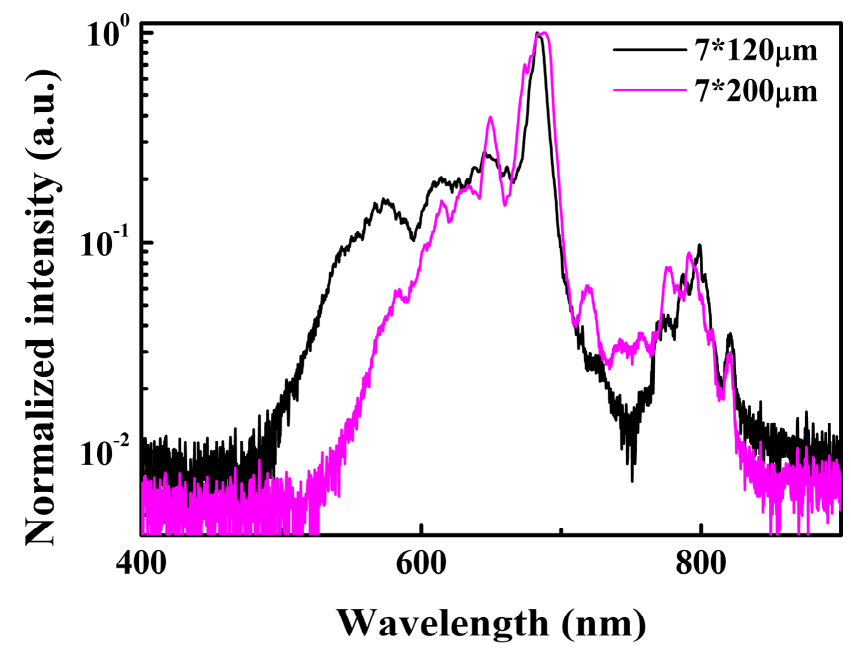

Figure 3. SC spectra after the laser beam propagated through the fused silica thin plates. Each spectrum was normalized by its maximum value.

Normally, the diameter of the spot gradually increased as the number of insetting plates increased, while the peak power density of the laser decreased. Therefore, it is advantageous to inset a thicker plate in the back. This can not only accumulate a greater effect of self-phase modulation but also avoid some adverse nonlinear influences, and it can finally contribute to further spectrum broadening. To provide a better comparison, the seventh thin plate was replaced with a $200 \mu \mathrm{m}$ thin plate based on the above experiments. It is necessary to adjust the position of the new inset plate to broaden the spectrum as much as possible. After passing through all the plates, the spectra were measured, as shown in Figure 4a. The spectra were broader than those of the seven plates with the same thickness, which extended to $470 \mathrm{~nm}$ in the short-wavelength region. In addition, the spectral intensity was significantly increased in the range from $470 \mathrm{~nm}$ to $650 \mathrm{~nm}$ compared to that of the seven $120 \mu \mathrm{m}$ plates. Figure $4 \mathrm{~b}$ shows a color photographic image of the beam profile in the far field that was taken by a digital camera. The most striking feature is a red ring surrounding a white circular central area.

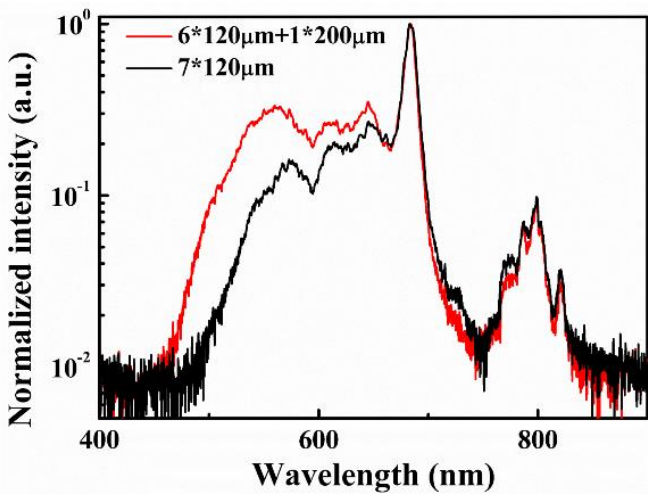

(a)

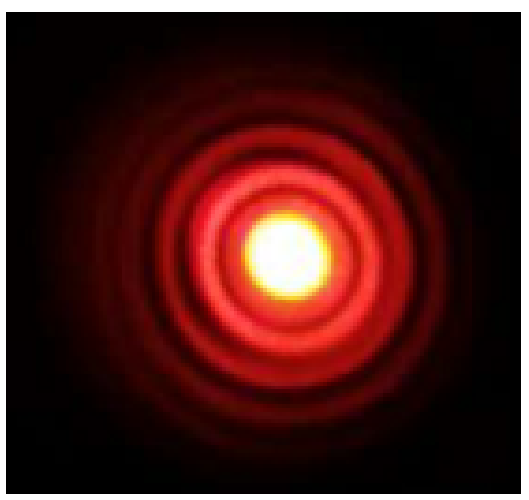

(b)

Figure 4. (a) SC spectrum after the laser beam propagated through a set of thin plates with mixed thickness. (b) The image of output beam profile.

We further analyzed the influence of mixed thin plates with different materials and thicknesses on spectral broadening. Usually, a thin plate of fused silica is used to generate SC. The material of the thin plates is not confined to fused silica. Because fluoride materials have a large bandgap, it is possible to further broaden the spectrum to the short-wavelength side. However, it is susceptible to optical damage owing to the low damage threshold of fluoride material [22]. We surmised that a plate with fluoride materials placed at the 
back of multiple thin plates would expand the spectrum to a greater degree. Then, we continued to insert a 1-mm-thick $\mathrm{CaF}_{2}$ plate ((111), Union Optic Inc., Wuhan, China) based on the broadened spectrum of the combined plates with different thicknesses. It should be noted that the $\mathrm{CaF}_{2}$ plate was placed at a distance of approximately $10 \mathrm{~cm}$ from the last fused silica plate to avoid optical damage. The position of the new inset plate was adjusted so that the spectrum was as broad as possible. The recorded spectra are shown in Figure 5. The spectrum was obviously broadened to approximately $430 \mathrm{~nm}$ in the short-wavelength region. Moreover, the intensity of short-wavelength components in the range of $430 \mathrm{~nm}$ to $600 \mathrm{~nm}$ was greatly increased. As a comparison, a 1-mm-thick fused silica plate was inserted. The spectrum no longer broadened, as shown by the green line in Figure 5. In fact, $\mathrm{CaF}_{2}$ had a higher nonlinear refractive index and lower critical power of self-focusing [23]. In terms of the results shown in Figure 2, more self-phase modulation might be accumulated through multiple thinner $\mathrm{CaF}_{2}$ plates as opposed to those that are 1-mm-thick. If there are thinner $\mathrm{CaF}_{2}$ plates, further broadening to wavelengths less than $400 \mathrm{~nm}$ could be achieved by inserting multiple $\mathrm{CaF}_{2}$ plates.

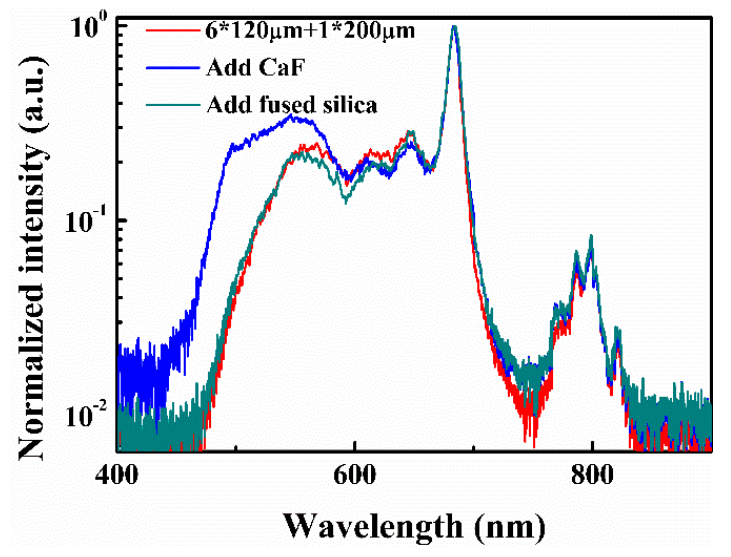

(a)

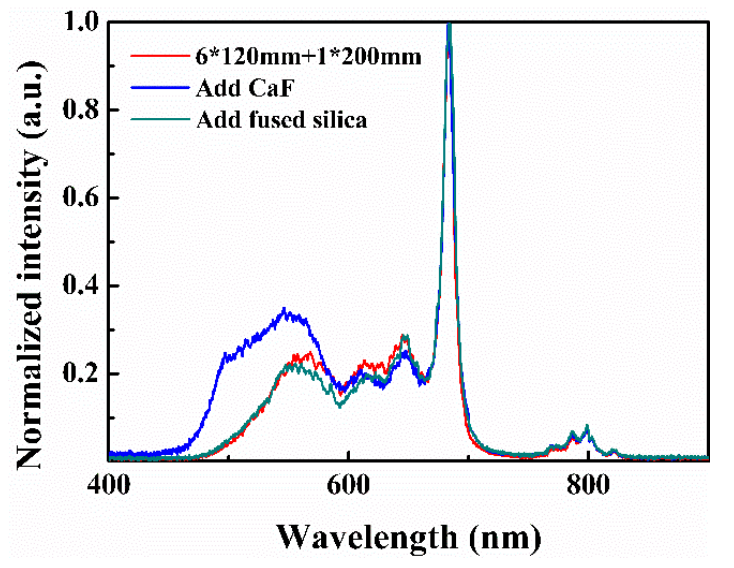

(b)

Figure 5. SC spectrum after the laser beam propagated through a set of mixed thin plates with different materials and thickness. (a) Spectra on a log scale; (b) Spectra on a linear scale.

The stability of the SC spectrum is an important factor in many applications. We investigated the stability of the SC spectrum in our experiment, which is crucial in transient absorption spectroscopy. In general, SC is generated by a single filament in bulk media in most experiments. Thus, a piece of fused silica with the size of $15 \mathrm{~mm} \times 15 \mathrm{~mm} \times 10 \mathrm{~mm}$ was used to induce a single filament for comparison. The spectra of SC obtained from the bulk fused silica and the mixed multiple thin plates are shown in Figure 6a. It was observed that the spectral intensity and broadening for the bulk fused silica was inferior to that for the mixed multiple thin plates. A color photographic image of the SC generated from the bulk fused silica was recorded by a digital camera and is shown in Figure 6b. The transverse section of the beam image reveals a typical conical emission pattern. A central white core was surrounded by colored Newton's rings appearing in an order opposite to diffraction. Furthermore, the spectral stability that we measured in multiple thin plates was compared to the case of a single filament generated in bulk fused silica. A series of spectra were collected by the spectrometer for 10 min with an acquisition interval time of $6 \mathrm{~s}$. The spectral integral intensity was calculated and normalized by its maximum value. The fluctuations of SC are represented by the difference between the normalized spectral integral intensity and its mean value. The spectral fluctuations with collection time are shown in Figure 7. The spectrum induced by a single filament exhibited small fluctuations, while, in the case of multiple thin plates, it was relatively stable. In addition, the standard deviation (SD) was used to quantitatively evaluate the stability of the SC spectrum. The SD was defined as $\sigma=\sqrt{\sum_{i=1}^{N}\left(I_{i}-\bar{I}\right)^{2} / N}$, where $N$ is the number 
of spectra collected, $I_{i}$ indicates each normalized spectral integral intensity, and $\bar{I}$ is the average value of the normalized spectral integral intensity. As the value of SD is smaller, the spectrum is more stable. The SDs of the spectra induced by bulk media, multiple thin plates, and the fundamental frequency light were calculated to be $0.0398,0.0285$, and 0.0149 , respectively. The results indicate that the spectrum induced by multiple thin plates shows better stability, which is 40 percent higher than that of the spectrum induced by a single filament in bulk media. The reason is as follows. As is well known, SC generation is a complex nonlinear optical process. The intensity stability of SC will be influenced by the nonlinear amplification of the input pulse fluctuations. In terms of the SC generation mechanism, the SC induced by the thin plates almost originated from pure self-phase modulation, while the SC induced by bulk media might be affected by many nonlinear optical processes besides the self-phase modulation. Thus, the stability of the SC generated from the multiple thin plates might be shot-noise-limited and better than that of the SC generated from bulk media.

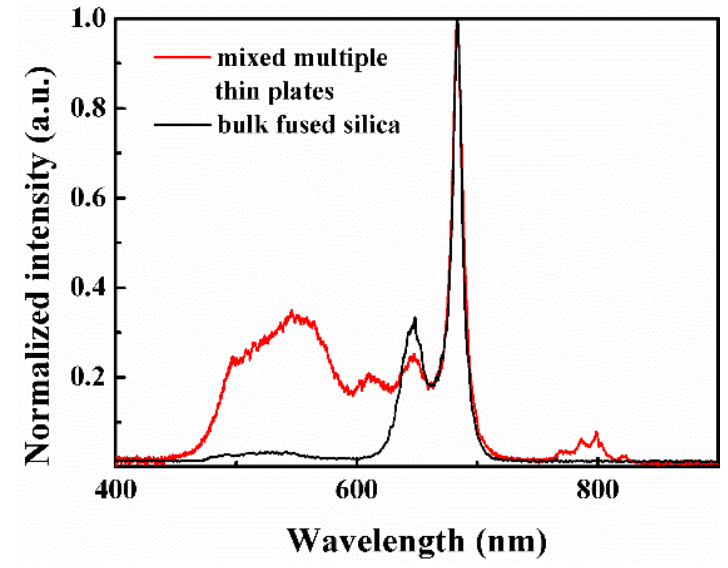

(a)

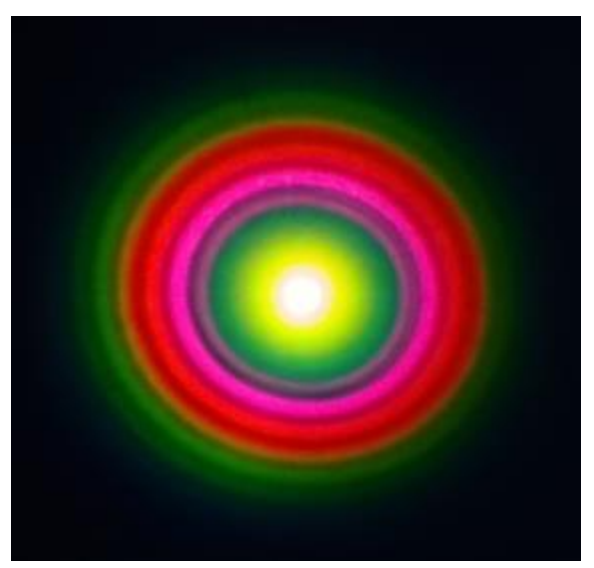

(b)

Figure 6. (a) SC spectra generated from the bulk fused silica and the mixed multiple thin plates. (b) Image of the SC beam profile in the bulk fused silica.

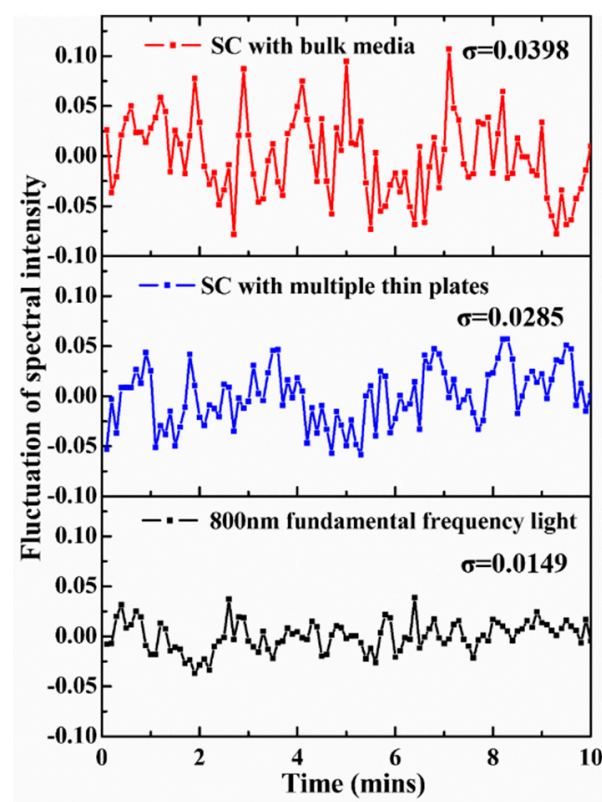

Figure 7. Fluctuation of normalized spectral integral intensity with collection time. $\sigma$ is the standard deviation of normalized spectral integral intensity. 


\section{Conclusions}

In summary, we have demonstrated a technique using multiple thin plates to generate a more intense and broader SC spectrum. This is achieved by a set of solid thin plates with different thicknesses and materials. The spectrum intensity and spectrum broadening in the short-wavelength region were significantly increased compared to those of traditional thin plates with identical thicknesses and materials. Furthermore, the spectrum exhibited excellent stability, which is superior to that of the spectrum induced by the single filament. Our results indicate that mixing multiple thin plates might be an interesting substitute for other SC generation techniques. For instance, when SC was generated in bulk material, the spectral broadening and intensity were insufficient. The results can be utilized in a wide range of applications, including femtosecond transient absorption spectroscopy, high-resolution investigation of ultrafast phenomena, and the generation of bright isolated attosecond pulses.

Author Contributions: Conceptualization, J.S. and W.T.; validation, J.S. and W.T.; investigation, J.L and Z.K.; resources, W.T.; writing—original draft preparation, J.L.; writing—review and editing, W.T. and J.L.; visualization, J.L.; supervision, X.H. All authors have read and agreed to the published version of the manuscript.

Funding: This research was funded by the National Natural Science Foundation of China, grant number 62027822 and 61690221; the National Key Research and Development Program of China, grant number 2019YFA0706402; the Natural Science Basic Research Plan in Shaanxi Province of China, grant number 2018JM6012, and the Fundamental Research Funds for the Central Universities, grant number xzy012019039.

Data Availability Statement: Data sharing is not applicable.

Conflicts of Interest: The authors declare no conflict of interest.

\section{References}

1. Alfano, P.R. The Supercontinuum Laser Source; Springer: New York, NY, USA, 1989.

2. Rothenberg, J.E. Space-time focusing: Breakdown of the slowly varying envelope approximation in the self-focusing of femtosecond pulses. Opt. Lett. 1992, 17, 1340-1342. [CrossRef] [PubMed]

3. Alfano, P.R. The Supercontinuum Laser Source: Fundamentals with Updated References, 2nd ed.; Springer: New York, NY, USA, 2006.

4. Ward, H.; Bergé, L. Temporal shaping of femtosecond solitary pulses in photoionized media. Phys. Rev. Lett. 2003, 90, 53901. [CrossRef] [PubMed]

5. Couairon, A.; Mysyrowicz, A. Femtosecond filamentation in transparent media. Phys. Rep. 2007, 411, 47-189. [CrossRef]

6. Bloembergen, N. The influence of electron plasma formation on superbroadening in light filaments. Opt. Commun. 1973, 8 , 285-288. [CrossRef]

7. Brabec, T.; Krauz, F. Intense few-cycle laser fields: Frontiers of nonlinear optics. Rev. Mod. Phys. 2000, 72, 545-591. [CrossRef]

8. Poudel, C.; Kaminski, C.F. Supercontinuum radiation in fluorescence microscopy and biomedical imaging applications. J. Opt. Soc. Am. B 2019, 36, 139-153. [CrossRef]

9. Petersen, C.R.; Moller, U.; Kubat, I.; Zhou, B.B.; Dupont, S.; Ramsay, J.; Benson, T.; Sujecki, S.; Abdel-Moneim, N.; Tang, Z.; et al. Mid-infrared supercontinuum covering the 1.4-13.3 $\mu \mathrm{m}$ molecular fingerprint region using ultra-high NA chalcogenide step-index fibre. Nat. Photonics 2014, 8, 830-834. [CrossRef]

10. Berge, L.; Rolle, J.; Kohler, C. Enhanced self-compression of mid-infrared laser filaments in argon. Phys. Rev. A 2013, 88, 023816. [CrossRef]

11. Cardin, V.; Thiré, N.; Beaulieu, S.; Wanie, V.; Légaré, F.; Schmidt, B.E. 0.42 TW 2-cycle pulses at $1.8 \mu \mathrm{m}$ via hollow-core fiber compression. Appl. Phys. Lett. 2015, 107, 181101. [CrossRef]

12. Nagy, T.; Hädrich, S.; Simon, P.; Blumenstein, A.; Walther, N.; Klas, R.; Buldt, J.; Stark, H.; Breitkopf, S.; Jójárt, P.; et al. Generation of three-cycle multi-millijoule laser pulses at $318 \mathrm{~W}$ average power. Optica 2019, 6, 1423-1424. [CrossRef]

13. Lu, C.H.; Tsou, Y.J.; Chen, H.Y.; Chen, B.H.; Cheng, Y.C.; Yang, S.D.; Chen, M.C.; Hsu, C.C.; Kung, A.H. Generation of intense supercontinuum in condensed media. Optica 2014, 1, 400-406. [CrossRef]

14. Cheng, Y.C.; Lu, C.H.; Lin, Y.Y.; Kung, A.H. Supercontinuum generation in a multi-plate medium. Opt. Express 2016, $24,7224-7231$. [CrossRef]

15. He, P.; Liu, Y.Y.; Zhao, K.; Teng, H.; He, X.K.; Huang, P.; Huang, H.D.; Zhong, S.Y.; Jiang, Y.J.; Fang, S.B.; et al. High-efficiency supercontinuum generation in solid thin plates at $0.1 \mathrm{TW}$ level. Opt. Lett. 2017, 42, 474-477. [CrossRef]

16. Lu, C.H.; Witting, T.; Husakou, A.; Vrakking, M.J.; Kung, A.H.; Furch, F.J. Sub-4 fs laser pulses at high average power and high repetition rate from an all-solid-state setup. Opt. Express 2018, 26, 8941-8956. [CrossRef] 
17. Canhota, M.; Weigand, R.; Crespo, H.M. Simultaneous measurement of two ultrashort near-ultraviolet pulses produced by a multiplate continuum using dual self-diffraction dispersion-scan. Opt. Lett. 2019, 44, 1015-1018. [CrossRef]

18. Lu, C.H.; Wu, W.H.; Kuo, S.H.; Guo, J.Y.; Chen, M.C.; Yang, S.D.; Kung, A.H. Greater than 50 times compression of 1030 nm $\mathrm{Yb}: \mathrm{KGW}$ laser pulses to single-cycle duration. Opt. Express 2019, 27, 15638-15648. [CrossRef] [PubMed]

19. Mourou, G.; Mironov, S.; Khazanov, E.; Sergeev, A. Single cycle thin film compressor opening the door to Zeptosecond-Exawatt physics. Eur. Phys. J. Spec. Top. 2014, 223, 1181-1188. [CrossRef]

20. Farinella, D.M.; Wheeler, J.; Hussein, A.E.; Nees, J.; Stanfield, M.; Beier, N.; Ma, Y.; Cojocaru, G.; Ungureanu, R.; Pittman, J.; et al. Focusability of laser pulses at petawatt transport intensities in thin-film compression. J. Opt. Soc. Am. B 2019, 36, A28-A32. [CrossRef]

21. Anashkina, E.A.; Ginzburg, V.N.; Kochetkov, A.A.; Yakovlev, I.V.; Kim, A.V.; Khazanov, E.A. Single-shot laser pulse reconstruction based on self-phase modulated spectra measurements. Sci. Rep. 2016, 6, 33749. [CrossRef] [PubMed]

22. Jia, T.Q.; Li, X.X.; Feng, D.H.; Cheng, C.F.; Li, R.X.; Chen, H.; Xu, Z.Z. Theoretical and experimental study on femtosecond laser induced damage in CaF2 crystals. Appl. Phys. A 2005, 81, 645-649. [CrossRef]

23. DeSalvo, R.; Sheik-Bahae, M.; Said, A.A.; Hagan, D.J.; Van Stryland, E.W. Z-scan measurements of the anisotropy of nonlinear refraction and absorption in crystals. Opt. Lett. 1993, 18, 194-196. [CrossRef] [PubMed] 